\title{
Determinants of Financing Constraints in East African Countries' SMEs
}

\author{
Alex Reuben Kira ${ }^{1}$ \\ ${ }^{1}$ Shanghai University of Finance and Economics, School of Finance, Shanghai, China \\ Correspondence: Alex Reuben Kira, Shanghai University of Finance and Economics, School of Finance, \#777 \\ Guoding Rd., Shanghai, China. E-mail: alexkira10@gmail.com
}

Received: January 14, 2013 Accepted: January 30, 2013 Online Published: March 13, 2013

doi:10.5539/ijbm.v8n8p49 URL: http://dx.doi.org/10.5539/ijbm.v8n8p49

\begin{abstract}
Small and Medium-sized Enterprises (SMEs) are the main player in developing and strengthening zonal integration. This study used a sample of 1933 firms located within 5 East African countries with economic integration known as East Africa Community (EAC). Data was extracted from World Bank Business Enterprises Survey to evaluate determinants of financing obstacles of firms. Analysis of data was conducted by running ordered probit model and multivariate regression model to determine which firms' industry or country's characteristics explain and experienced financial constraints in their operations. The study found out that financing constraints in East African firms broadly based across firms but SMEs are mostly affected.
\end{abstract}

Keywords: East African countries, financing constraint, SMEs, ordered probit model

\section{Introduction}

Globally SME sector has been reporting difficulties in access to finance (Bebczuk, 2004; Slotty, 2009; Balling et al., 2009; Irwing \& Scott, 2010; Yongqian et al., 2012). Access to external finance to SMEs has become more costly and troublesome while their accessibility has done sharply declined. SMEs' financing constraints limit their investment opportunities and stagnant growth. Access to finance is widely perceived to be an essential factor for firms, and especially SMEs, to maintain their daily business operation as well as to achieve long-term investment opportunities and development targets. Presence of general limitations on access to capital markets, many East African firms heavily rely on the banking sector for credit. Therefore, a well-functioning banking sector plays an important role in channeling resources to the best firms and investment ventures. Financing constraints crucially limit firm's growth, availability of productive resources resulting to sluggish of a sector which might pose threat to the sector's contribution to the economy.

Recently SMEs financing has raised interest in policymakers, researchers and practitioners as an agenda of interest on how to encounter the problem. The purpose of this study is to evaluate the nature of the financial difficulties encountered by East African area firms in their operations and to draw a portrait of East African area firms under financing constraints. The study uses survey data from the World Bank Business Enterprises Survey to establish how East African firms face difficulties with access to finance. The aim is to provide an extensive understanding on how East African firms assessed their access external finances to invest in different business opportunities. The study evaluates the problem by analyzing country characteristics and individual firm financing constraints actual experiences on the problem. Particularly, two indicators were developed for evaluation of attributes impact financing constraints in East African SMEs'. The first indicator is based on the country's factors such classification of industrial sector (i.e. manufacturing, service, construction) and ownership (i.e. locally owned, foreign ownership or state ownership) ranking of problems faced by firms; the second one is based on firms' individual characteristics in getting access to external finance.

The study found out that financial constraints were broadly based across firms than country wise. The financial constraint tends severely to harm more small and young. The study suggest that the strategic interest of East African banks in the SME segment can make an important contribution to closing the "SME financing gap" in the region compared to other developing countries. He study concludes that this trend should be supported and encouraged through reforms to soften the negative impact of those obstacles which are hindering the further involvement of banks with SMEs. This paper is organized as follows: section 2 briefly reviews the related literature on SMEs' access to finance; section 3 provides a description of the data and introduces the empirical 
methodology then section 4 which describes study results and the final section is a conclusion of the study.

\section{Literature Review}

\subsection{SMEs Overview and Its Contributions}

SMEs play a significant part in economic growth worldwide including in African countries (Note 1). Studies point out that in developed and developing economies SMEs contribute on average $60 \%$ of formal employment in the manufacturing sector (Ayyagari et al., 2007). In Africa, SME sector's total contribution in job creation in manufacturing is about three-quarters (Ayyagari et al., 2007). An important aspect for SME sector development is access to finance particularly from financial institutions. Le, Venkatesh and Nguyen (2006) pointed out that the achievement stage for any particular SME is to have adequate access to external sources of finance. Firm-level data collected by the World Bank show that shortage on access to finance perceived to be one of the main obstacles to doing business (Note 2). Several studies have revealed that financing is a major constraint for SMEs to grow than for larger firms, mainly in the developing world (Beck et al., 2005; Beck et al., 2006; Fatoki \& Assah, 2011, Kira \& He, 2012).

SMEs thought to have a financial growth cycle in which financial needs and options change as the business grows, gains further experience, and become less informational opaque. Figure 1 shows this in a stylized fashion in which firms' trend on size/age/information availability in access to financing sources. Smaller/younger/more opaque firms lie near the left end of the growth trend line indicating that they must rely on initial insider finance, trade credit, and/or angel finance (Note 3). Initial financing include funds provided by the owner(s), family members and friends prior to and at the time of the firm's commencement of operation. As firms grow, they gain access to intermediate finance on the equity side [venture capital (Note 5)] and on the debt side (banks, finance companies, etc.). In the long run, if the firm remain in operation and continue to grow might gain access to debt markets and stock market. Firm's growth cycle model might not become applicable to all small businesses since firm size, age, and information availability are far from perfectly correlated. The model also demonstrates some features at different points which stimulate funding to firm occasionally. Figure 1 explain the general idea on how sources of finance become important in the financing growth cycle as well as the points in the cycle at which different types of financing are attained as the firm grow. For example, even the very largest firms obtain funding through bank loans or private placements, but this is not shown in the figure.

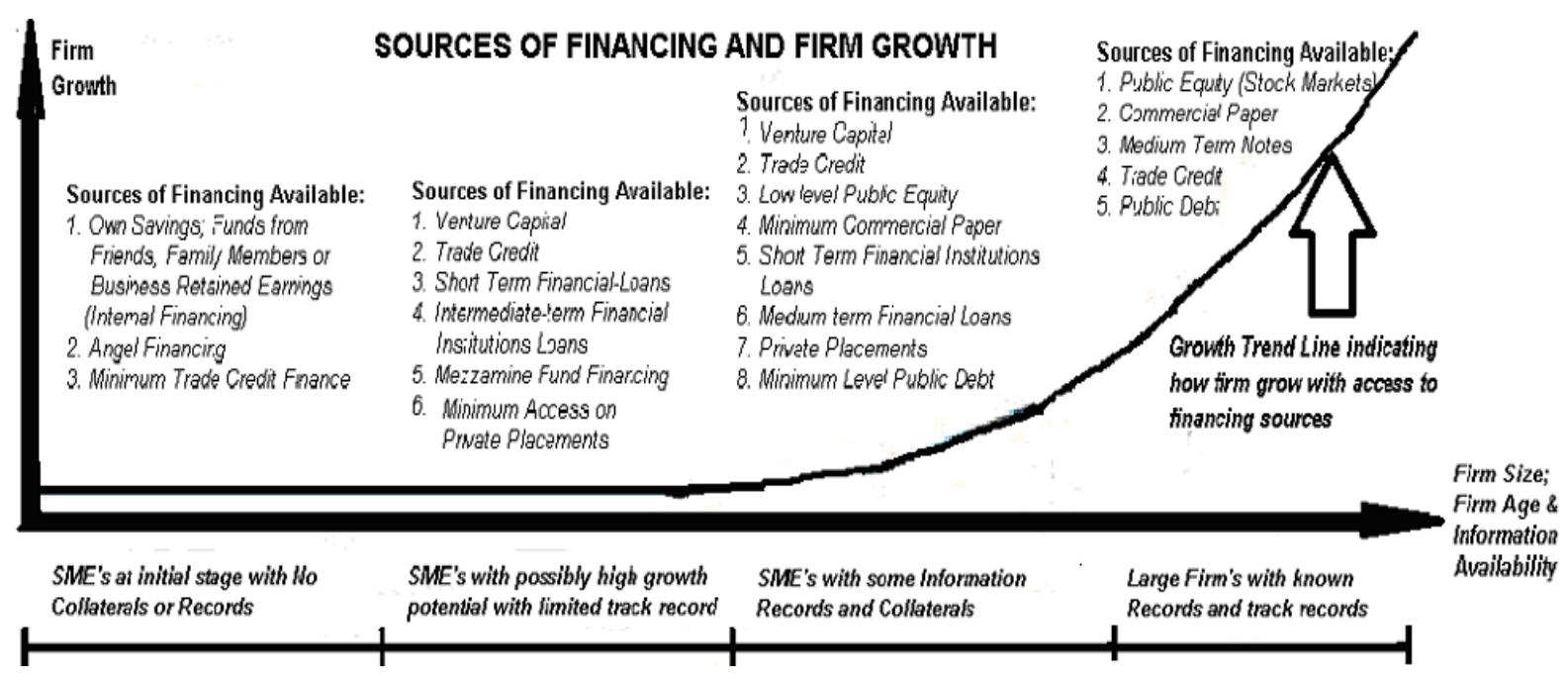

Figure 1. Firm growth and access to financing

Conservative viewers argue that bank or commercial finance company lending would typically not be available to SMEs until they achieve a level of production where their balance sheets reflect substantial tangible business assets that might be pledged as collateral such as inventory, equipments and accounts receivable (Note 6). This sequencing of funding over the growth cycle of a firm can be viewed in the context of the modem information-based theory of security design and the notion of a financial pecking order. Costly state verification (Townsend 1979, Diamond 1984) and adverse selection (Myers 1984, Myers and Majluf 1984, Nachman \& Noe, 1994) arguments suggest the optimality of debt contracts after insider finance has been exhausted that firm has to 
search for external sources such as debt and equity to enjoy the benefits on utilization of external debt (Modigliani \& Miller, 1963). These debt contracts could include trade credit, loans from commercial bank and finance companies. Conversely, moral hazard can make debt contracts quite problematic. Moral hazard problems are likely to occur when the amount of external finance needed is large relative to the amount of insider finance (inclusive of any personal wealth at risk via pledges of personal collateral or guarantees). This suggests that external equity finance specifically angel and venture capital may be particularly important when these conditions hold and the moral hazard problem is acute. The fact that high-growth, high-risk new ventures often obtain angel finance and/or venture capital before they obtain significant amounts of external debt finance suggests that the moral hazard problem may be particularly acute for these firms (Note 7).

Most of international development communities have programmed SME access to finance as an important policy priority (Note 8). Global economic integration is changing the competitive paradigm in which all businesses operation requiring a competitive strategy to positively impact long-term growth and survival. The small business sector has become more important as they emerge as a dominant force impacting the growth of national economies (Shridhar, 2006). Recently, SMEs empowerment takes a prominent position in the development agenda of most countries this is due to their contribution to a vibrant and growing industrial sector. Most countries create appropriate institutional environment that recognizes SMEs to take advantages attached in employment creation, poverty alleviation as well as facilitating economic growth.

In Tanzania, SMEs contributions to GDP estimated to be ranked about one third (Note 9). It is also estimated about $20 \%$ of the labor force in Tanzania which is almost 3 million people are employed in small businesses, in which of these are micro enterprises consisted of 1.7 million businesses operating in the informal sector alone. SME sector play a major role in economic development of Tanzanian economy. The International Finance Company (Note 10) of the World Bank estimates that there are approximately 2.7 million enterprises in exist in Tanzania whereby over $98 \%$ are SMEs.

In Burundi, Most Burundian businesses admitted that access to credit is a serious constraint (Note 11). However, while the demand for credit is real and pervasive, there are constraints on it. Most small businesses (especially individual entrepreneurs) finance their initial operations primarily with their own funds and capital. Many of these entrepreneurs are suspicious of formal credit and would rather rely solely on their own resources and those of family and friends. Small Burundian businesses often have a foot in the informal economy and are usually trying to minimize their exposure to scrutiny from the state.

In the case of Kenya, the SME sector plays a crucial role to the Kenyan economy. SMEs employed about 5.1 million people representing $74 \%$ of the total national employment and also contribute about $88 \%$ of the total job creation at any one time; they also contribute in the Gross Domestic Product of the country, whereby they contribute about $24.5 \%$ to the GDP (Maina, 2006). Atieno (2009) pointed out that the development of SMEs has been identified as one of the strategies for generating industrialization, employment generation and poverty reduction in Kenya. Atieno (2009) reported governments' objective has been outlined in Kenya's major policy documents such as the Sessional Paper Number 2 of 1996 on Industrial Transformation to the year 2020, and Sessional Paper Number 2 of 2005 on the Development of MSEs for Employment and Wealth Creation.

In Rwanda, over $90.8 \%$ of Rwanda's workforce is employed in the private sector (Note 12) which makes it a catalytic sector in terms of reforms to ensure inclusive growth. Over 123,000 SMEs operate in the private sector, accounting for $98 \%$ of all businesses and $84 \%$ of private sector employment. However, $88 \%$ of these SMEs are informal and as such, their contribution to total tax revenues, estimated at less than 2\% in Financial Year 2009/10, remains insufficient. Moreover, improvements in investment climate are yet to translate into private sector development (Note 13).

In Uganda; the SMEs represent a significant part of the economy. There are special linkages promotion programs that are used to promote SMEs sector to keep a fast-track vehicle for creating a dynamic SME sector. SMEs comprise over $90 \%$ of the private sector in Uganda representing a very significant role in stimulating the economic growth of the country (Kuzilwa, 2005). Ugandan SMEs create productive employment; provide a good source of the tax revenues for social as well as economic development and they also increase the export revenues vis-à-vis import substitution and thus balancing the terms of trade. SMEs contribute about $75 \%$ of the GDP and employment of approximately 2.5 million people in Uganda. SMEs contribute about $75 \%$ of the GDP and employment of approximately 2.5 million people in Uganda (African Development Bank, 2011). MMA consulting company operating in East African countries revealed the following information relating to investment opportunities in East African countries by 2010.

Figure 2 extracted from Match Maker Associates Ltd (MMA) investment focus reviews is a region, MMA is 
Tanzania-based consulting company. Through their work in the East African agribusiness sector they identified attractive investment opportunities with high social and environmental impact they revealed that lack of finance is one of constraint limiting business operations in the region even though business opportunities exist. The consulting company revealed that the majority of the East African population is growing and depends on the SME sector. Today, SMEs operate in every corner of the East Africa and have great potential in job creation as well as generation of a widespread economic benefit.

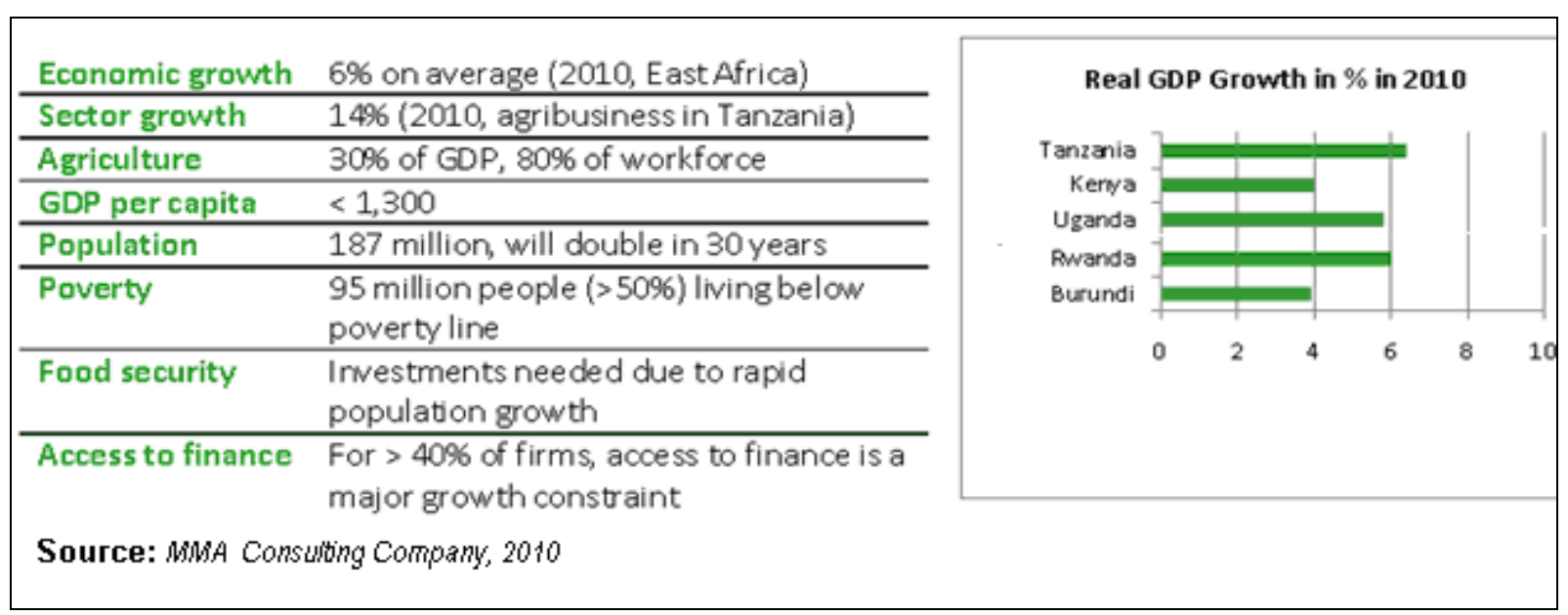

Figure 2. East African countries GDP, growth, agriculture; population, \& access to finance

\subsection{SMEs Access to External Financing}

Access to finance is reported to be a severe problem for SMEs in Africa. Unavailability of finance to SME sector has been viewed as a critical element for the development of SMEs. Levy (1993) highlighted the consequences of limited access to financial resources by SMEs. Normally SMEs face higher transaction costs than larger enterprises in obtaining credit (Saito and Villanueva, 1981) and availability of funds to finance working capital (Peel and Wilson, 1996). In addition, information asymmetries associated with lending to small scale borrowers have continued to restrict the flow of finance to SMEs.

Regardless of SMEs being considered as a major driver of innovation and employment, as well as their potentiality in a country's economic development; recent empirical studies have refined this view, suggesting that the future of SME sector might be jeopardized due financial constraints (Beck et al, 2005; Aghion et al., 2007, Fatoki \& Assah, 2011). SME sector are commonly more being financially constrained and experience financial difficulties in access to external finance. Literature has revealed an existence and the determinants of financing constraints being extensive very large based on two major hypothetical thoughts: asymmetric information and agency costs. Fazzari et al., (1988) pointed out that the existence of financial constraints is due to the presence of asymmetric information while internal and external capitals are not perfect substitutes (Note 14). Consequently, firms' investment opportunities might depend on financial aspects such as the availability of internal financing, access to new debt or equity finance or the functioning of particular credit markets.

Empirical literature strengthens that external financing is more costly to SMEs which led them to rely on internal sources of finance to fund their investments because of asymmetric information problem and the agency costs existence which cement their financing constraint problem. These problems are believed to be severely impacted SMEs because of the following reasons: one, Size, SMEs size is small compared to large firms which might influence the quality and the quantity of information available on their records as well as collateral. Smaller firms are often perceived to be more informational opaque than larger firms and monitoring cost weight more heavily on smaller-scale projects (Devereux \& Schiantarelli, 1989; Gilchrist \& Himmelberg, 1991; Berger \& Udell, 1988; Beck et al., 2005). Furthermore, most of SMEs during start-up are observed to encounter more informational opaque problem and agency costs because they are new as well as time limits their track record and reputation to access external finance (European Communities, 2009; Irwin \& Scot, 2010; Oreoluwa, 2011). Finally, because of the prevailing problems led SMEs to depend more on bank financing than larger enterprises as they cannot raise funds from stock markets since stock market require more relevant and transparent 
information to attract investors. Whited and $\mathrm{Wu}$ (2006) study found out that mostly financially constrained firms are small because they have low analyst coverage which affect their bond rating. Whited and $\mathrm{Wu}(2006)$ evidenced that small firms are more financially constrained since they lack bond rating and also possess low analyst coverage in the US.

Several related recent studies have acknowledged a number of determinants of access to finance: Fatoki and Assah (2011) suggested SMEs have to own tangible assets, maintain proper business information and improve their management skills to accelerate access of debt financing from lenders. Colluzi et al. (2009) found out that young and small firms are significantly facing financial constraints in their study on the significance of firm characteristics on access to external finance. Atanasova and Wilson (2004) proposed that firm's total asset collateral is an essential determinant to access credit. Beck et al. (2006) uncover that countries with higher levels of financial intermediary development, more efficient legal systems, higher GDP-per-capita and more liquid stock market report lower financing obstacles. The study which was conducted UK manufacturing firm between 1989 and 1999 by Bougheas et al. (2006), noticed several firm characteristics including collateral, age, profitability, riskiness and size do influence accessibility of debt financing. Harrison and McMillan (2003) evidenced that listed firms and foreign owned firms encounter financial constraints compared to unlisted and locally firms. An industrial sector in which a firm conducts business does play an influential role in determining accessibility to external capital markets (Hall et al., 2000). Sectors which require huge capital intensive to operate such as manufacturing and construction seems to attract investors/lenders to extend capital financing. Canton et al., (2010) found out that firm's age, firm-bank relationship, and banking sector degree of competition are the determinants of firm's perceived financial constraints in banking industry at the European Union level. The survey study of determinants of finance access to SMEs in ECB and the European Commission resulted that firm's ownership structure and age are vital determinants of the perceived financial constraints regardless in which industry firm operate or the firm size Ferrando and Griesshaber (2011).

This study intends to provide relevant determinants of SMEs financial constraints by evaluating the firm's characteristic and the country's characteristics. Additionally, econometric model using multivariate and ordered probit models will be consolidated to establish the financial constraint relationship between firm and country levels to unveil the underlying assumption is that countries and firms facing better financial services accelerate their growth and contribution to the country's economic development.

\section{Research Methodology}

\subsection{Source of Data}

This study uses the World Bank's Business Enterprise Survey (Note 15) data to evaluate SMEs financial constraint in the East African Countries (i.e., Burundi, Kenya, Rwanda, Tanzania, and Uganda). The five countries belong to the East African Community with a long term objective of forming a federation. The sample is consistently defined in all countries and includes the entire manufacturing sector, construction sectors and the services sector. This study excludes public utilities, government services, health care and financial services sectors from the sample. The surveys collect a wide array of qualitative and quantitative information through face-to-face interviews with firm managers and owners regarding the business environment in their countries and the productivity of their firms. Several topics are covered during the survey and include: the obstacles to doing business, infrastructure, labor, corruption, law and order, regulation, finance, innovation and technology, trade, and firm productivity which in totality effect business environment. The qualitative and quantitative data collected through the surveys connect a country's business environment characteristic with firm productivity and performance. The study uses the data collected between 2002 and 2007 by World Bank whereby over 10,000 firms around the world were surveyed including Burundi, Kenya, Rwanda, Tanzania and Uganda data. The definition of indicators can be found on the database website. The sample constitutes 242 firms from Burundi, 657 firms from Kenya, 158 firms from Rwanda, 368 firms from Tanzania, and 508 firms from Uganda declared they have financial constraint. This study evaluated the financial constraints existence in East African countries' business environments which impacts on the availability of financial resources to SMEs. The study suggests policies to improve availability of financial resources to SMEs in East Africa. The study uses both cross tabular analysis and multivariate analysis of variance to achieve the study objectives.

\subsection{Data Characteristics and Methodological Approach}

The main purpose of the survey is to qualify firms' access to finance in the East African Countries. The survey covered a wide area of East African business environment traits but for this study focus is only firms' attributes and country's characteristics on the financing constraints. It contains some information on firms' and country's characteristics such firm size (small, medium, large); Ownership (local private owned, foreign private owned, or 
state owned); Age (in years of operation); industrial type (Manufacturing, Service, Construction), and form of business organization (Public limited company, private held company, sole proprietorship, partnership or other forms of incorporation). The sampling method was performed as the resulting sample is representative across several dimensions, i.e. for each of the East African countries (i.e. Burundi, Kenya, Rwanda, Tanzania and Uganda), across firm size [i.e. micro, small, medium and large firms] and their main industrial sector.

Table 1. Composition of sample report

\begin{tabular}{clcc}
\hline & Variables & No. of Observations & Percent \\
\hline \multirow{3}{*}{ Industrial Sector } & Manufacturing & 1025 & 53.00 \\
& Service & 454 & 23.47 \\
& Construction & 454 & 23.47 \\
& Small & 1213 & 62.75 \\
& Medium & 555 & 28.70 \\
& Large & 165 & 8.53 \\
& Public Ltd Company & 12 & 0.62 \\
& Private Ltd Company & 701 & 36.26 \\
\cline { 2 - 2 } Incorporation & Sole Proprietorship & 920 & 47.57 \\
& Partnership & 278 & 14.37 \\
& Other & 21 & 1.09 \\
& Less than 5 yrs & 377 & 19.49 \\
& From 5 to 9 yrs & 608 & 31.45 \\
& From 10 to 19 yrs & 580 & 29.99 \\
& From 20 to 49 yrs & 336 & 17.37 \\
& Above 50 yrs & 32 & 1.65 \\
& Private (Local) & 1687 & 87.23 \\
& Private (Foreign) & 219 & 11.32 \\
Ownership & State/Government & 16 & 0.83 \\
& Other & 11 & 0.57
\end{tabular}

Source: Author's calculations based on WBES data.

Table 1 report the composition of the study's sample according to country, size, legal status, (incorporation), industrial type, ownership and age as the firms' characteristics selected for the 1933 firms responding to the survey questionnaire that they have financial constraint. Small firms employ less than 19 employees, Medium firms employ 20 to 99 employees and large firms employ over 100 employees. Legal status represents incorporation of a firm: Publicly listed companies are those firms listed in stock markets; Private held limited companies are those companies which are legal and separate entities but are not listed at stock market. Sole proprietorship and partnership are forms of business organizations without separate legal entity. Other legal entities represent all other forms of business organization which not fit in public listed company, privately held company, sole proprietorship or partnership such as cooperatives, non governmental organizations (NGO), religious business operations etc. Industry type classifies business operation as either manufacturing, service or construction sector. Ownership defines modal of ownership within a firm: Foreign or Local ownership demonstrates that firm owners are foreigners or locals respectively. Firms were interviewed whether they face financial constraints. Management of surveyed firms was asked to rate the financial constraints encountered by their firms during their daily operations by quantifying financial obstacle ranging from 1 , minor obstacle and 5 , very severe obstacle. Surveyed questions rate the impact of financial constraints on their firms' growth. The questionnaires narrate the financial constraints firms encounter from financial institutions included are: collateral requirements, application procedures and complexity, high interest rates, credit availability or accessibility, need for special connection or corruption to access credit and the size of the credit and maturity insufficiency. 
Table 2. Summary statistics

\begin{tabular}{|c|c|c|c|c|c|c|}
\hline & Variables & Observation & Mean & Std. Deviation & Minimum & Taximum \\
\hline \multirow{4}{*}{$\begin{array}{l}\text { Industrial } \\
\text { Sector }\end{array}$} & Manufacturing & 1933 & 0.5303 & 0.49921 & 0.00 & 1.00 \\
\hline & Service & 1933 & 0.2349 & 0.42403 & 0.00 & 1.00 \\
\hline & Construction & 1933 & 0.2349 & 0.42403 & 0.00 & 1.00 \\
\hline & Small & 1933 & 0.6275 & 0.48359 & 0.00 & 1.00 \\
\hline \multirow[t]{4}{*}{ Size } & Medium & 1933 & 0.2871 & 0.45253 & 0.00 & 1.00 \\
\hline & Large & 1933 & 0.0854 & 0.27949 & 0.00 & 1.00 \\
\hline & Public Ltd Company & 1933 & 0.0062 & 0.07857 & 0.00 & 1.00 \\
\hline & Private Ltd Company & 1933 & 0.3626 & 0.48089 & 0.00 & 1.00 \\
\hline \multirow[t]{5}{*}{ Incorporation } & Sole Proprietorship & 1933 & 0.4759 & 0.49955 & 0.00 & 1.00 \\
\hline & Partnership & 1933 & 0.1438 & 0.35100 & 0.00 & 1.00 \\
\hline & Other & 1933 & 0.0109 & 0.10369 & 0.00 & 1.00 \\
\hline & Less than 5 yrs & 1933 & 0.1950 & 0.39633 & 0.00 & 1.00 \\
\hline & From 5 to $9 \mathrm{yrs}$ & 1933 & 0.3145 & 0.46445 & 0.00 & 1.00 \\
\hline \multirow[t]{4}{*}{ Age } & From 10 to $19 \mathrm{yrs}$ & 1933 & 0.3001 & 0.45840 & 0.00 & 1.00 \\
\hline & From 20 to $49 \mathrm{yrs}$ & 1933 & 0.1738 & 0.37906 & 0.00 & 1.00 \\
\hline & Above 50 yrs & 1933 & 0.0166 & 0.12763 & 0.00 & 1.00 \\
\hline & Private (Local) & 1933 & 0.8727 & 0.33335 & 0.00 & 1.00 \\
\hline \multirow{3}{*}{ Ownership } & Private (Foreign) & 1933 & 0.1133 & 0.31704 & 0.00 & 1.00 \\
\hline & State/Government & 1933 & 0.0083 & 0.09063 & 0.00 & 1.00 \\
\hline & Other & 1933 & 0.0057 & 0.07524 & 0.00 & 1.00 \\
\hline \multirow{7}{*}{$\begin{array}{c}\text { Financing } \\
\text { Constraints }\end{array}$} & General & 1700 & 3.4149 & 1.33422 & 1.00 & 5.00 \\
\hline & Collateral & 1834 & 4.2742 & 1.19538 & 1.00 & 5.00 \\
\hline & Application Procedures & 1473 & 2.9881 & 1.51813 & 1.00 & 5.00 \\
\hline & High Interest rates & 1253 & 2.0817 & 1.34308 & 1.00 & 5.00 \\
\hline & Credit & 1798 & 4.0253 & 1.27440 & 1.00 & 5.00 \\
\hline & Corruption\& Special Connections & 1503 & 2.7807 & 1.33534 & 1.00 & 5.00 \\
\hline & Loan Size \& Maturity & 1506 & 2.2711 & 1.37674 & 1.00 & 5.00 \\
\hline
\end{tabular}

Source: Author's calculations based on WBES data.

Table 3a. Correlation matrix

\begin{tabular}{|c|c|c|c|c|c|c|c|c|c|}
\hline & Gcfin & Manuf & Service & Const & Small & Medium & Large & Plc & Pltdcoy \\
\hline Gcfin & 1.000 & & & & & & & & \\
\hline Manuf & $\begin{array}{l}0.064^{* *} \\
(0.005)\end{array}$ & 1.000 & & & & & & & \\
\hline Service & $\begin{array}{c}0.032 \\
(0.164)\end{array}$ & $\begin{array}{l}-0.589^{* *} \\
(0.000)\end{array}$ & 1.000 & & & & & & \\
\hline Const & $\begin{array}{l}-0.106^{* *} \\
(0.000)\end{array}$ & $\begin{array}{l}-0.589^{* *} \\
(0.000)\end{array}$ & $\begin{array}{l}-0.307^{* *} \\
(0.000)\end{array}$ & 1.000 & & & & & \\
\hline Small & $\begin{array}{c}0.243 \\
(0.058)\end{array}$ & $\begin{array}{l}-0.251^{* *} \\
(0.000)\end{array}$ & $\begin{array}{l}0.230^{* *} \\
(0.000)\end{array}$ & $\begin{array}{l}0.066^{* *} \\
(0.004)\end{array}$ & 1.000 & & & & \\
\hline
\end{tabular}




\begin{tabular}{|c|c|c|c|c|c|c|c|c|c|}
\hline \multirow{2}{*}{ Medium } & 0.302 & $0.144^{* *}$ & $0.149^{* *}$ & -0.020 & $-0.824^{* *}$ & 1.000 & & & \\
\hline & $(0.002)$ & $(0.000)$ & $(0.000)$ & $(0.384)$ & 0.000 & & & & \\
\hline \multirow{2}{*}{ Large } & $-0.071^{* *}$ & $0.202^{* *}$ & $0.156^{* *}$ & $0.082^{* *}$ & $-0.397^{* *}$ & $-0.194^{* *}$ & 1.000 & & \\
\hline & $(0.002)$ & $(0.000)$ & $(0.000)$ & $(0.000)$ & $(0.000)$ & $(0.000)$ & & & \\
\hline \multirow{2}{*}{ Plc } & $-0.049^{*}$ & $0.048^{*}$ & -0.028 & -0.028 & $-0.075^{* *}$ & 0.008 & $0.117^{* *}$ & 1.000 & \\
\hline & $(0.030)$ & $(0.035)$ & $(0.214)$ & $(0.214)$ & $(0.001)$ & $(0.723)$ & $(0.000)$ & & \\
\hline \multirow{2}{*}{ Pltdcoy } & $-0.115^{* *}$ & $0.259^{* *}$ & $-0.179^{* *}$ & $-0.126^{* *}$ & $-0.394^{* *}$ & $0.240^{* *}$ & $0.293^{* *}$ & $-0.060^{* *}$ & 1.000 \\
\hline & $(0.000)$ & $(0.000)$ & $(0.000)$ & $(0.000)$ & $(0.000)$ & $(0.000)$ & $(0.000)$ & $(0.009)$ & \\
\hline \multirow{2}{*}{ Solepro } & $0.071^{* *}$ & $-0.288^{* *}$ & $0.183^{* *}$ & $0.156^{* *}$ & $0.359^{* *}$ & $-0.236^{* *}$ & $-0.239^{* *}$ & $-0.075^{* *}$ & $-0.719^{* *}$ \\
\hline & $(0.002)$ & $(0.000)$ & $(0.000)$ & $(0.000)$ & $(0.000)$ & $(0.000)$ & $(0.000)$ & $(0.001)$ & $(0.000)$ \\
\hline \multirow{2}{*}{ Partnerp } & $0.069^{* *}$ & 0.031 & -0.001 & -0.036 & $0.060^{* *}$ & -0.003 & $-0.099^{* *}$ & -0.032 & $-0.309^{* *}$ \\
\hline & $(0.002)$ & $(0.169)$ & $(0.964)$ & $(0.116)$ & $(0.009)$ & $(0.907)$ & $(0.000)$ & $(0.155)$ & $(0.000)$ \\
\hline \multirow{2}{*}{ Other } & 0.001 & 0.039 & -0.023 & -0.023 & $-0.053^{*}$ & 0.033 & 0.039 & -0.008 & $-0.079^{* *}$ \\
\hline & $(0.962)$ & $(0.089)$ & $(0.318)$ & $(0.318)$ & $(0.019)$ & $(0.150)$ & $(0.083)$ & $(0.716)$ & $(0.001)$ \\
\hline \multirow{2}{*}{ Age $1-4$ years } & 0.235 & $-0.204^{* *}$ & $0.137^{* *}$ & $0.103^{* *}$ & $0.217^{* *}$ & $-0.159^{* *}$ & $-0.118^{* *}$ & -0.039 & $-0.195^{* *}$ \\
\hline & $(0.025)$ & $(0.000)$ & $(0.000)$ & $(0.000)$ & $(0.000)$ & $(0.000)$ & $(0.000)$ & $(0.087)$ & $(0.000)$ \\
\hline \multirow{2}{*}{ Age 5- 9 years } & 0.137 & $-0.050^{*}$ & $0.058^{*}$ & 0.001 & $0.093^{* *}$ & -0.041 & $-0.095^{* *}$ & -0.025 & $-0.112^{* *}$ \\
\hline & $(0.010)$ & $(0.028)$ & $(0.010)$ & $(0.982)$ & $(0.000)$ & $(0.073)$ & $(0.000)$ & $(0.269)$ & $(0.000)$ \\
\hline \multirow{2}{*}{$\begin{array}{l}\text { Age } 10-19 \\
\text { years }\end{array}$} & -0.020 & 0.028 & $-0.072^{* *}$ & 0.039 & -0.037 & $0.049^{*}$ & -0.014 & -0.009 & 0.037 \\
\hline & $(0.379)$ & $(0.216)$ & $(0.001)$ & $(0.084)$ & $(0.101)$ & $(0.033)$ & $(0.533)$ & $(0.704)$ & $(0.106)$ \\
\hline \multirow{2}{*}{$\begin{array}{l}\text { Age } 20-49 \\
\text { years }\end{array}$} & -0.035 & $0.210^{* *}$ & $-0.109^{* *}$ & $-0.138^{* *}$ & $-0.271^{* *}$ & $0.155^{* *}$ & $0.217^{* *}$ & $0.051^{*}$ & $0.259^{* *}$ \\
\hline & $(0.122)$ & $(0.000)$ & $(0.000)$ & $(0.000)$ & $(0.000)$ & $(0.000)$ & $(0.000)$ & $(0.026)$ & $(0.000)$ \\
\hline \multirow{2}{*}{$\begin{array}{c}\text { Age above } 50 \\
\text { years }\end{array}$} & -0.043 & $0.090^{* *}$ & $-0.053^{*}$ & $-0.053^{*}$ & $-0.076^{* *}$ & 0.007 & $0.120^{* *}$ & $0.093^{* *}$ & $0.113^{* *}$ \\
\hline & $(0.056)$ & $(0.000)$ & $(0.020)$ & $(0.020)$ & $(0.001)$ & $(0.749)$ & $(0.000)$ & $(0.000)$ & $(0.000)$ \\
\hline \multirow{2}{*}{ PvtLC } & $0.054^{*}$ & $-0.067^{* *}$ & $0.061^{* *}$ & 0.017 & $0.194^{* *}$ & $-0.118^{* *}$ & $-0.144^{* *}$ & $-0.167^{* *}$ & $-0.193^{* *}$ \\
\hline & $(0.018)$ & $(0.003)$ & $(0.007)$ & $(0.442)$ & $(0.000)$ & $(0.000)$ & $(0.000)$ & $(0.000)$ & $(0.000)$ \\
\hline \multirow{2}{*}{ PvtFG } & -0.044 & $0.058^{*}$ & $-0.056^{*}$ & -0.013 & $-0.177^{* *}$ & $0.116^{* *}$ & $0.119^{* *}$ & $0.117^{* *}$ & $0.209^{* *}$ \\
\hline & $(0.054)$ & $(0.010)$ & $(0.015)$ & $(0.561)$ & $(0.000)$ & $(0.000)$ & $(0.000)$ & $(0.000)$ & $(0.000)$ \\
\hline \multirow{2}{*}{ State } & -0.028 & $0.052^{*}$ & -0.037 & -0.024 & $-0.107^{* *}$ & 0.030 & $0.136^{* *}$ & $0.211^{* *}$ & -0.010 \\
\hline & $(0.212)$ & $(0.023)$ & $(0.103)$ & $(0.298)$ & $(0.000)$ & $(0.182)$ & $(0.000)$ & $(0.000)$ & $(0.675)$ \\
\hline \multirow{2}{*}{ Other1 } & -0.018 & -0.011 & 0.007 & 0.007 & 0.016 & -0.002 & -0.023 & -0.006 & -0.014 \\
\hline & $(0.419)$ & $(0.614)$ & $(0.767)$ & $(0.767)$ & $(0.493)$ & $(0.916)$ & $(0.310)$ & $(0.793)$ & $(0.534)$ \\
\hline \multirow{2}{*}{ FcColl } & $0.199^{* *}$ & $0.232^{* *}$ & $-0.143^{* *}$ & $-0.129^{* *}$ & $-0.064^{* *}$ & 0.112 & $0.052^{*}$ & -0.018 & $0.087^{* *}$ \\
\hline & $(0.000)$ & $(0.000)$ & $(0.000)$ & $(0.000)$ & $(0.005)$ & $(0.036)$ & $(0.021)$ & $(0.426)$ & $(0.000)$ \\
\hline \multirow{2}{*}{ FcApp } & $0.072^{* *}$ & $0.114^{* *}$ & $-0.078^{* *}$ & $-0.056^{*}$ & $-0.062^{* *}$ & 0.560 & $0.085^{* *}$ & 0.549 & 0.329 \\
\hline & $(0.001)$ & $(0.000)$ & $(0.001)$ & $(0.014)$ & $(0.007)$ & $(0.013)$ & $(0.000)$ & $(0.014)$ & $(0.022)$ \\
\hline \multirow{2}{*}{ FcHInte } & $0.123^{* *}$ & $0.090^{* *}$ & $-0.073^{* *}$ & -0.033 & -0.014 & -0.018 & $0.054^{*}$ & -0.015 & -0.003 \\
\hline & $(0.000)$ & $(0.000)$ & $(0.001)$ & $(0.149)$ & $(0.525)$ & $(0.424)$ & $(0.017)$ & $(0.521)$ & (0.908) \\
\hline \multirow{2}{*}{ FcCredit } & 0.342 & $-0.054^{*}$ & 0.043 & 0.022 & -0.018 & 0.025 & -0.009 & 0.009 & $0.068^{* *}$ \\
\hline & $(0.007)$ & $(0.017)$ & $(0.061)$ & $(0.344)$ & $(0.422)$ & $(0.271)$ & $(0.693)$ & $(0.700)$ & $(0.003)$ \\
\hline \multirow{2}{*}{ FcConr } & $0.057^{*}$ & $0.062^{* *}$ & $0.084^{* *}$ & 0.010 & $0.066^{* *}$ & 0.043 & $0.046^{*}$ & -0.007 & $0.055^{*}$ \\
\hline & $(0.012)$ & $(0.006)$ & $(0.000)$ & $(0.647)$ & $(0.003)$ & $(0.061)$ & $(0.043)$ & $(0.767)$ & $(0.015)$ \\
\hline \multirow{2}{*}{ FcSizMat } & 0.634 & 0.018 & $0.058^{*}$ & $-0.079^{* *}$ & $-0.155^{* *}$ & $0.107^{* *}$ & $0.096^{* *}$ & -0.001 & $0.127^{* *}$ \\
\hline & $(0.011)$ & $(0.424)$ & $(0.011)$ & $(0.001)$ & $(0.000)$ & $(0.000)$ & $(0.000)$ & $(0.958)$ & $(0.000)$ \\
\hline
\end{tabular}


Table 3b. Correlation matrix

\begin{tabular}{|c|c|c|c|c|c|c|c|c|}
\hline & Solepro & Partnerp & Other & Age_5to9 & Age_5 & Age_10to19 & Age_20to49 & Age_50 \\
\hline Solepro & 1.000 & & & & & & & \\
\hline \multirow[t]{2}{*}{ Partnerp } & $-0.391^{* *}$ & 1.000 & & & & & & \\
\hline & $(0.000)$ & & & & & & & \\
\hline \multirow[t]{2}{*}{ Other } & $-0.100^{* *}$ & -0.043 & 1.000 & & & & & \\
\hline & $(0.000)$ & $(0.059)$ & & & & & & \\
\hline \multirow[t]{2}{*}{ Age_5to9 } & $0.097^{* *}$ & 0.037 & $-0.049^{*}$ & 1.000 & & & & \\
\hline & $(0.000)$ & $(0.107)$ & $(0.030)$ & & & & & \\
\hline \multirow[t]{2}{*}{ Age_5 } & $0.190^{* *}$ & 0.018 & -0.039 & $-0.333^{* *}$ & 1.000 & & & \\
\hline & $(0.000)$ & $(0.434)$ & $(0.087)$ & $(0.000)$ & & & & \\
\hline \multirow[t]{2}{*}{ Age_10to19 } & -0.039 & -0.005 & 0.029 & $-0.444^{* *}$ & $-0.322^{* *}$ & 1.000 & & \\
\hline & $(0.090)$ & $(0.842)$ & $(0.196)$ & $(0.000)$ & $(0.000)$ & & & \\
\hline \multirow[t]{2}{*}{ Age_20to49 } & $-0.235^{* *}$ & $-0.048^{*}$ & $0.057^{*}$ & $-0.311^{* *}$ & $-0.226^{* *}$ & $-0.300^{* *}$ & 1.000 & \\
\hline & $(0.000)$ & $(0.035)$ & $(0.012)$ & $(0.000)$ & $(0.000)$ & $(0.000)$ & & \\
\hline \multirow[t]{2}{*}{ Age_50 } & $-0.107^{* *}$ & -0.030 & 0.026 & $-0.088^{* *}$ & $-0.064^{* *}$ & $-0.085^{* *}$ & $-0.060^{* *}$ & 1.000 \\
\hline & $(0.000)$ & $(0.186)$ & $(0.262)$ & $(0.000)$ & $(0.005)$ & $(0.000)$ & $(0.009)$ & \\
\hline \multirow[t]{2}{*}{ PvtLC } & $0.209^{* *}$ & $0.055^{*}$ & $-0.155^{* *}$ & 0.035 & $0.090^{* *}$ & -0.011 & $-0.099^{* *}$ & $-0.072^{* *}$ \\
\hline & $(0.000)$ & $(0.016)$ & $(0.000)$ & $(0.127)$ & $(0.000)$ & $(0.635)$ & $(0.000)$ & $(0.002)$ \\
\hline \multirow[t]{2}{*}{ PvtFG } & $-0.190^{* *}$ & $-0.049^{*}$ & 0.026 & -0.017 & $-0.073^{* *}$ & 0.005 & $0.069^{* *}$ & $0.069^{* *}$ \\
\hline & $(0.000)$ & $(0.032)$ & $(0.262)$ & $(0.451)$ & $(0.001)$ & $(0.840)$ & $(0.003)$ & $(0.002)$ \\
\hline \multirow[t]{2}{*}{ State } & $-0.087^{* *}$ & -0.021 & $0.376^{* *}$ & $-0.062^{* *}$ & $-0.045^{*}$ & 0.002 & $0.124^{* *}$ & -0.012 \\
\hline & $(0.000)$ & $(0.352)$ & $(0.000)$ & $(0.006)$ & $(0.048)$ & $(0.913)$ & $(0.000)$ & $(0.602)$ \\
\hline \multirow[t]{2}{*}{ Other1 } & -0.017 & -0.011 & $0.125^{* *}$ & -0.007 & -0.037 & 0.026 & 0.002 & 0.044 \\
\hline & $(0.455)$ & $(0.616)$ & $(0.000)$ & $(0.765)$ & $(0.102)$ & $(0.262)$ & $(0.944)$ & $(0.053)$ \\
\hline \multirow[t]{2}{*}{ FcColl } & $0.075^{* *}$ & -0.015 & 0.018 & 0.000 & $-.089^{* *}$ & $.049^{*}$ & 0.034 & -0.003 \\
\hline & $(0.001)$ & $(0.508)$ & $(0.436)$ & $(0.990)$ & $(0.000)$ & $(0.031)$ & $(0.134)$ & $(0.908)$ \\
\hline \multirow[t]{2}{*}{ FcApp } & 0.322 & 0.011 & -0.042 & -0.027 & 0.006 & -0.039 & $0.067^{* *}$ & 0.025 \\
\hline & $(0.002)$ & $(0.629)$ & $(0.065)$ & $(0.236)$ & $(0.806)$ & $(0.083)$ & $(0.003)$ & $(0.271)$ \\
\hline \multirow[t]{2}{*}{ FcHInte } & 0.546 & -0.002 & -0.032 & -0.028 & $0.046^{*}$ & -0.033 & 0.019 & 0.022 \\
\hline & $(0.036)$ & $(0.934)$ & $(0.155)$ & $(0.219)$ & $(0.044)$ & $(0.145)$ & $(0.408)$ & $(0.327)$ \\
\hline \multirow[t]{2}{*}{ FcCredit } & $0.095^{* *}$ & 0.044 & -0.010 & -0.018 & -0.008 & -0.020 & $0.047^{*}$ & 0.023 \\
\hline & $(0.000)$ & $(0.054)$ & $(0.663)$ & $(0.433)$ & $(0.734)$ & $(0.377)$ & $(0.041)$ & $(0.315)$ \\
\hline \multirow[t]{2}{*}{ FcConr } & $0.086^{* *}$ & $0.062^{* *}$ & $-0.046^{*}$ & $-0.045^{*}$ & 0.021 & -0.034 & $0.059^{* *}$ & 0.043 \\
\hline & $(0.000)$ & $(0.007)$ & $(0.042)$ & $(0.049)$ & $(0.351)$ & $(0.139)$ & $(0.009)$ & $(0.061)$ \\
\hline \multirow[t]{2}{*}{ FcSizMat } & $0.149^{* *}$ & $0.052^{*}$ & $-0.046^{*}$ & $-0.066^{* *}$ & -0.001 & $-0.046^{*}$ & $0.122^{* *}$ & $0.048^{*}$ \\
\hline & $(0.000)$ & $(0.022)$ & $(0.043)$ & $(0.004)$ & $(0.960)$ & $(0.043)$ & $(0.000)$ & $(0.034)$ \\
\hline
\end{tabular}

Source: Author's calculations based on WBES data. 
Table 3c. Correlation matrix

\begin{tabular}{ccccccccccc}
\hline & PvtLC & PvtFG & State & Other1 & FcColl & FcApp & FcHInte & FcCred & FcConr & FcSizMat \\
\hline PvtLC & 1.000 & & & & & & & & & \\
PvtFG & $0.636^{* *}$ & 1.000 & & & & & & & & \\
& $(0.000)$ & & & & & & & & & \\
State & $-0.239^{* *}$ & -0.033 & 1.000 & & & & & & & \\
& $(0.000)$ & $(0.151)$ & & & & & & & & \\
Other1 & $-0.198^{* *}$ & -0.027 & -0.007 & 1.000 & & & & & & \\
& $(0.000)$ & $(0.235)$ & $(0.761)$ & & & & & & & \\
FcColl & 0.547 & 0.007 & 0.003 & 0.029 & 1.000 & & & & & \\
& $(0.014)$ & $(0.766)$ & $(0.898)$ & $(0.207)$ & & & & & & \\
FcApp & 0.164 & $0.048^{*}$ & -0.041 & -0.013 & -0.027 & 1.000 & & & & \\
& $(0.032)$ & $(0.035)$ & $(0.074)$ & $(0.568)$ & $(0.235)$ & & & & & \\
FcHInte & 0.413 & -0.017 & -0.018 & 0.011 & $0.113^{* *}$ & $0.158^{* *}$ & 1.000 & & & \\
& $(0.019)$ & $(0.458)$ & $(0.421)$ & $(0.636)$ & $(0.000)$ & $(0.000)$ & & & & \\
FcCredit & 0.369 & 0.033 & $-0.051^{*}$ & 0.015 & $-0.131^{* *}$ & $-0.097^{* *}$ & 0.037 & 1.000 & & \\
& $(0.020)$ & $(0.152)$ & $(0.025)$ & $(0.519)$ & $(0.000)$ & $(0.000)$ & $(0.102)$ & & & \\
FcConr & $0.048^{*}$ & $0.064^{* *}$ & $-0.053^{*}$ & 0.007 & -0.038 & $0.203^{* *}$ & $0.102^{* *}$ & $0.266^{* *}$ & 1.000 & \\
& $(0.036)$ & $(0.005)$ & $(0.019)$ & $(0.749)$ & $(0.099)$ & $(0.000)$ & $(0.000)$ & $(0.000)$ & & \\
FcSizMat & $0.095^{* *}$ & $0.111^{* *}$ & -0.030 & -0.010 & -0.041 & $0.141^{* *}$ & 0.032 & $0.149^{* *}$ & $0.440^{* *}$ & 1.000 \\
& $(0.000)$ & $(0.000)$ & $(0.181)$ & $(0.663)$ & $(0.073)$ & $(0.000)$ & $(0.160)$ & $(0.000)$ & $(0.000)$ & \\
\hline
\end{tabular}

Notes: **. Correlation is significant at the 0.01 level (2-tailed).

*. Correlation is significant at the 0.05 level (2-tailed).

Source: Author's calculations based on WBES data.

Table 3a-c shows the correlation coefficients or matrix between the general financial obstacle and different firm characteristics. SMEs report significantly higher financing obstacles than large firms. The correlation coefficients show the non existence of high correlation among the variables. The multicollinearity problem was also evaluated among variable and since no correlation coefficient is greater than 0.90 , therefore the problem none exist as per the Field (2005) theory $(r>0.90)$.

\section{Multivariate Analysis and Results}

The simple statistics presented in table 2 demonstrates that there are significant relationship between firm characteristics and financing obstacles. A multivariate analysis was adopted to analyze the relationship exist between firm financing obstacles and firm characteristics within East African countries through the following equation:

$$
\text { GFCons }_{i, k}=f(F C, C)+E_{i, k}=\beta \text { Firm Charactreristics }_{i, k}+\text { Country }_{k}+\varepsilon_{i, k}
$$

Where: GFCons (General Financing Constraint); Firm financing obstacle is either general financing obstacle or one of the specific obstacles encountered by firm $i$ in country $k$. FC(Firm Characteristics) is a vector of firm's features which include the firm size dummies, incorporation dummies, industrial sector dummy variables, ownership dummy variables (locally, foreign or government/state ownership) and firm age dummies. Country (C) is a vector of country dummies that influence undetected country's specific factors that might influence firm's response on financing constraints.

Probit model and multivariate regressions were used to estimate regression (1) to establish which firms' or country's characteristics have influence financing constraints on their operations. The study assumes the disturbance parameter has a normal distribution and use standard maximum likelihood estimation. In applying the model clustered error terms is tolerable since omitted country specific characteristics might cause error terms to be correlated to firms within countries. 
The study also evaluates whether other institutional factors, financial factors as well as economic development factors help to alleviate financing obstacles. Country dummies were replaced with country-level variables. The regression estimated to evaluate the situation is:

General Financing Constraint $=\alpha+$ Industrial sector + Size + Ownership + Age + Incorporation $+\varepsilon$

General Financing Constraint $=\alpha+\beta_{1}$ Manufacturing $+\beta_{2}$ Services $+\beta_{3}$ Private Local Ownership $+\beta_{4}$ Small $+\beta_{5}$ Medium +

$\beta_{6}$ Soleproprietorship $1+\beta_{7}$ Partnership $+\beta_{8}$ Age $(1-4)+\beta_{9}$ Age $(5-9)+\varepsilon$

Table 4. Regression on financing constraints and firm characteristics results

\begin{tabular}{|c|c|c|}
\hline Variables & General Financing Constraint (1) & General Financing Constraint (2) \\
\hline Manufacturing & $\begin{array}{c}0.272 \\
(0.000)\end{array}$ & $\begin{array}{c}0.366 \\
(0.000)\end{array}$ \\
\hline Service & $\begin{array}{c}0.233 \\
(0.001)\end{array}$ & $\begin{array}{c}0.211 \\
(0.003)\end{array}$ \\
\hline Construction & $\begin{array}{l}-0.259 \\
(0.000)\end{array}$ & $\begin{array}{l}-0.254 \\
(0.000)\end{array}$ \\
\hline Private Local Ownership & $\begin{array}{c}0.186 \\
(0.010)\end{array}$ & $\begin{array}{c}0.186 \\
(0.010)\end{array}$ \\
\hline Private Foreign Ownership & $\begin{array}{l}-0.176 \\
(0.021)\end{array}$ & $\begin{array}{l}-0.176 \\
(0.021)\end{array}$ \\
\hline Small & & $\begin{array}{c}0.311 \\
(0.000)\end{array}$ \\
\hline Medium & & $\begin{array}{c}0.234 \\
(0.012)\end{array}$ \\
\hline Large & & $\begin{array}{l}-0.282 \\
(0.001)\end{array}$ \\
\hline Public Ltd Company & & $\begin{array}{l}-0.632 \\
(0.040)\end{array}$ \\
\hline Private Ltd Company & & $\begin{array}{l}-0.269 \\
(0.000)\end{array}$ \\
\hline Sole Proprietorship & & $\begin{array}{c}0.170 \\
(0.001)\end{array}$ \\
\hline Partnership & & $\begin{array}{c}0.200 \\
(0.004)\end{array}$ \\
\hline Age 0 - 4 years & & $\begin{array}{c}0.425 \\
(0.029)\end{array}$ \\
\hline Age 5 - 9 years & & $\begin{array}{c}0.413 \\
(0.032)\end{array}$ \\
\hline Age $10-19$ years & & $\begin{array}{l}-0.114 \\
(0.041)\end{array}$ \\
\hline Age 20 - 49 years & & $\begin{array}{l}-0.154 \\
(0.022)\end{array}$ \\
\hline Age 50 and above years & & $\begin{array}{l}-0.417 \\
(0.028)\end{array}$ \\
\hline $\begin{array}{l}\text { Cut } 1 \\
\text { Constant }\end{array}$ & $\begin{array}{c}0.991 \\
(0.000)\end{array}$ & $\begin{array}{l}1.186 \\
(0.000)\end{array}$ \\
\hline Cut 2 & 0.728 & 0.835 \\
\hline Constant & $(0.000)$ & $(0.000)$ \\
\hline Cut 3 & 0.717 & 0.904 \\
\hline Constant & $(0.000)$ & $(0.000)$ \\
\hline Log-likelihood & 678.576 & 1380.829 \\
\hline LR-Chi-square & 78.097 & 117.058 \\
\hline $\mathrm{R}^{2}$ - P-value & 0.040 & 0.059 \\
\hline
\end{tabular}

Source: Author's calculations based on WBES data. 


\subsection{Research Results and Analysis}

The General financing constraint is the response from firms on how financing constraint affects their firm's operations and growth. Responses vary from 1 (No obstacle), 2 (Minor obstacle), 3 (moderate obstacle), 4 (Major obstacle) and 5 (very severe obstacle). The regressions are estimated with ordered probit model. The results evidently show that there is a linear relationship existence between financing constraints and firms' characteristics. Firm's industrial sector, size, incorporation, age and ownership are likely to experience financing constraints however; all categories are not always significantly different from each another.

The industrial sector result implies that financing constraint is positively significantly related. Manufacturing and service sector are the one demonstrated financing constraints. The regressions in column (1) and (2) in Table 4 indicate that manufacturing and services are the strongest predictors of financing constraints. The study includes country and sectoral dummies in all regressions to evaluate how country's and firm's specific characteristics might influence firms' response in the study. Manufacturing sector comprises agriculture and natural resources including Food, Wood, Wood Products and Furniture; Nonmetallic minerals; Manufacturing Industries such as Textile; Machinery and equipments; Chemicals; Metal and Metal Products; and Other Manufacturing. Service sector comprises Services and Retail business including Garments; Retail; Transport, Knowledge Based Industries such as Electronics and Information Technology while Construction Sector stand itself.

Firm size influence firm's financing constraint whereby small and medium firms are more affected. This study used the dummy variables for small, medium and large as indicators of firm size. The results supported Kira and He (2012), Artola and Genre (2011), Fatoki and Assah (2011) that significantly financing constraints suffered severely to younger firms than large firms. The study concluded that SMEs are more likely to experience financial constraints than large firms.

The study finds out that local privately owned firms report higher financing obstacles than firm privately foreign owned. Under incorporation, firms which operate as a sole proprietorship or partnership are mostly to experience financing obstacles than Private Limited firms or Public Listed firms (PLC). The form of business organization (incorporation) does influence financing access to finance, access to capital in which has an impact in financing obstacle of the firm (Cassar, 2004; Abor, 2008, Kira \& He, 2012). This study concluded that the form of business organization has an effect on equity - debt decisions which impact financing constraints on SMEs.

Firm aged less than 10 years ( 1 to 4 years and 5 to 9 years) were resulted to experience financing constraints in East African countries. Firms aged less than 10 years are significantly more prone to financing constraints than mature firms (i.e. more than 10 years old), but, according to this study, the model reveal that very young firms (i.e. less than five years old) are not significantly different from firms aged 5 to 9. Cassar (2004) supported the results that younger firm face heavily financial problem associated with information asymmetry and informational opaque (Devereux \& Schiantarelli, 1989; Gilchrist \& Himmelberg, 1991; Berger \& Udell, 1988; Beck et al., 2005, Artola \& Genre, 2011).

Table 5. Quantifying the effect of financing constraints and firm characteristics

\begin{tabular}{lcc}
\hline Variables & General Financing Constraint (1) & General Financing Constraint (2) \\
\hline Manufacturing & 0.5303 & 0.5600 \\
& 0.4697 & 0.4400 \\
Service & 0.0605 & 0.1200 \\
& 0.7651 & 0.5573 \\
Construction & 0.2349 & 0.4427 \\
& 0.5303 & 0.1145 \\
Private Local Ownership & 0.2349 & 0.4824 \\
& 0.7651 & 0.5176 \\
& -0.5303 & -0.0352 \\
& 0.8727 & 0.5507 \\
& 0.1273 & 0.4493 \\
\hline
\end{tabular}




\begin{tabular}{|c|c|c|}
\hline \multirow[t]{3}{*}{ Private Foreign Ownership } & 0.1133 & 0.4840 \\
\hline & 0.8867 & 0.5160 \\
\hline & -0.7734 & -0.0320 \\
\hline \multirow[t]{3}{*}{ Small } & & 0.5631 \\
\hline & & 0.4369 \\
\hline & & 0.1261 \\
\hline \multirow[t]{3}{*}{ Medium } & & 0.5351 \\
\hline & & 0.4649 \\
\hline & & 0.0703 \\
\hline \multirow[t]{3}{*}{ Large } & & 0.4000 \\
\hline & & 0.6000 \\
\hline & & -0.2000 \\
\hline \multirow[t]{3}{*}{ Public Ltd Company } & & 0.2500 \\
\hline & & 0.7500 \\
\hline & & -0.5000 \\
\hline \multirow[t]{3}{*}{ Private Ltd Company } & & 0.4565 \\
\hline & & 0.5435 \\
\hline & & -0.0870 \\
\hline \multirow[t]{3}{*}{ Sole Proprietorship } & & 0.5783 \\
\hline & & 0.4217 \\
\hline & & 0.1565 \\
\hline \multirow[t]{3}{*}{ Partnership } & & 0.6367 \\
\hline & & 0.3633 \\
\hline & & 0.2734 \\
\hline \multirow[t]{3}{*}{$0-4$ years } & & 0.5889 \\
\hline & & 0.4111 \\
\hline & & 0.1777 \\
\hline \multirow[t]{3}{*}{$5-9$ years } & & 0.5543 \\
\hline & & 0.4457 \\
\hline & & 0.1086 \\
\hline \multirow[t]{3}{*}{$10-19$ years } & & 0.5276 \\
\hline & & 0.4724 \\
\hline & & 0.0552 \\
\hline \multirow[t]{3}{*}{20 - 49 years } & & 0.5060 \\
\hline & & 0.4941 \\
\hline & & 0.0119 \\
\hline \multirow[t]{3}{*}{50 and above years } & & 0.3438 \\
\hline & & 0.6563 \\
\hline & & -0.3125 \\
\hline
\end{tabular}

Source: Author's calculations based on WBES data.

Table 5 shows the economic significance of firm characteristics for their financing obstacles, this study report the 
estimated probability that a firm describes financing as a major obstacle depending on its characteristics. Based on the regressions in table 4, estimated probabilities of rating financing obstacles as a major obstacle to the operation and growth of the firm (Financing obstacle $=5$ ) are presented to establish the strength of a specific variable. Probability estimations were calculated for each firm's variable at its actual value to quantify the problem's strength. On the case of dummies, the first row reports the probability if the dummy variable takes on the value one while the second row reports the probability if the dummy variable takes on the value zero while the third row reports the difference between the first and second row to quantify the variables' effect. In the overall sample, manufacturing, private local ownership, service, small and medium enterprises, sole proprietorship and partnership, firm aged (1-4) years and (5-9) years each explain more than $5 \%$ difference in the probability that a firm describes financing as a major obstacle. The results show how the problem can be quantified across the firms and evidence the spread of the problem across East African firms.

Table 6. Specific financing obstacles and firm characteristics

\begin{tabular}{|c|c|c|c|c|c|c|}
\hline Variables & $\begin{array}{l}\text { Financing } \\
\text { Constraint } \\
\text { Collateral }\end{array}$ & $\begin{array}{l}\text { Financing } \\
\text { Constraint } \\
\text { Application } \\
\text { Procedures }\end{array}$ & $\begin{array}{c}\text { Financing } \\
\text { Constraint } \\
\text { High Interest } \\
\text { Rates }\end{array}$ & $\begin{array}{c}\text { Financing } \\
\text { Constraint } \\
\text { Credit } \\
\text { Accessibility }\end{array}$ & $\begin{array}{c}\text { Financing } \\
\text { Constraint Special } \\
\text { connections \& } \\
\text { Corruption }\end{array}$ & $\begin{array}{c}\text { Financing } \\
\text { Constraint } \\
\text { Loan Size \& } \\
\text { Maturity }\end{array}$ \\
\hline \multirow{2}{*}{ Manufacturing } & 0.582 & 0.182 & 0.086 & 0.731 & 0.184 & 0.087 \\
\hline & $(0.000)$ & $(0.000)$ & $(0.019)$ & $(0.014)$ & $(0.000)$ & $(0.010)$ \\
\hline \multirow{2}{*}{ Service } & 0.383 & 0.167 & 0.111 & 0.623 & 0.172 & 0.244 \\
\hline & $(0.000)$ & $(0.000)$ & $(0.011)$ & $(0.023)$ & $(0.000)$ & $(0.049)$ \\
\hline \multirow{2}{*}{ Construction } & -0.350 & -0.077 & -0.008 & 0.012 & 0.069 & -0.170 \\
\hline & $(0.000)$ & $(0.042)$ & $(0.849)$ & $(0.791)$ & $(0.144)$ & $(0.000)$ \\
\hline \multirow{2}{*}{$\begin{array}{l}\text { Private Local } \\
\text { Ownership }\end{array}$} & 0.880 & 0.226 & 0.299 & 0.665 & 0.657 & 0.183 \\
\hline & $(0.013)$ & $(0.025)$ & $(0.046)$ & $(0.026)$ & $(0.027)$ & $(0.001)$ \\
\hline \multirow{2}{*}{$\begin{array}{l}\text { Private Foreign } \\
\text { Ownership }\end{array}$} & -0.035 & 0.092 & -0.065 & 0.055 & 0.044 & 0.212 \\
\hline & $(0.696)$ & $(0.069)$ & $(0.252)$ & $(0.398)$ & $(0.501)$ & $(0.000)$ \\
\hline \multirow{2}{*}{ Small } & 0.334 & 0.775 & 0.907 & 0.877 & 0.936 & 0.234 \\
\hline & $(0.000)$ & $(0.027)$ & $(0.004)$ & $(0.006)$ & $(0.003)$ & $(0.000)$ \\
\hline \multirow{2}{*}{ Medium } & 0.228 & 0.255 & 0.260 & 0.567 & 0.944 & 0.174 \\
\hline & $(0.000)$ & $(0.011)$ & 0.044 & 0.025 & $(0.033)$ & $(0.000)$ \\
\hline \multirow{2}{*}{ Large } & 0.371 & 0.191 & -0.120 & -0.051 & 0.032 & 0.205 \\
\hline & $(0.092)$ & $(0.059)$ & $(0.082)$ & $(0.479)$ & $(0.673)$ & $(0.001)$ \\
\hline \multirow{2}{*}{ Public Ltd Company } & -0.425 & 0.161 & -0.117 & 0.151 & -0.120 & -0.026 \\
\hline & $(0.201)$ & $(0.408)$ & $(0.625)$ & $(0.553)$ & $(0.621)$ & $(0.910)$ \\
\hline \multirow{2}{*}{ Private Ltd Company } & 0.262 & 0.047 & -0.012 & 0.111 & 0.004 & 0.191 \\
\hline & $(0.000)$ & $(0.175)$ & $(0.742)$ & $(0.009)$ & $(0.922)$ & $(0.000)$ \\
\hline \multirow{2}{*}{ Sole Proprietorship } & 0.201 & 0.233 & 0.279 & -0.134 & 0.404 & 0.205 \\
\hline & $(0.000)$ & $(0.039)$ & $(0.032)$ & $(0.001)$ & $(0.035)$ & $(0.000)$ \\
\hline \multirow{2}{*}{ Partnership } & 0.363 & 0.291 & 0.550 & 0.069 & 0.079 & 0.189 \\
\hline & $(0.043)$ & $(0.005)$ & $(0.030)$ & $(0.230)$ & $(0.172)$ & $(0.029)$ \\
\hline \multirow{2}{*}{ Age 0 - 4 years } & 0.206 & 0.228 & 0.097 & 0.824 & 0.037 & 0.116 \\
\hline & $(0.003)$ & $(0.016)$ & $(0.012)$ & $(0.038)$ & $(0.062)$ & $(0.018)$ \\
\hline \multirow{2}{*}{ Age 5 - 9 years } & 0.333 & 0.526 & -0.043 & 0.389 & -0.025 & 0.092 \\
\hline & $(0.038)$ & $(0.026)$ & $(0.256)$ & $(0.082)$ & $(0.560)$ & $(0.025)$ \\
\hline
\end{tabular}




\begin{tabular}{lcccccc}
\hline Age 10 - 19 years & 0.217 & -0.278 & -0.050 & -0.015 & 0.005 & -0.060 \\
& $(0.077)$ & $(0.074)$ & $(0.199)$ & $(0.725)$ & $(0.912)$ & $(0.146)$ \\
Age 20 - 49 years & 0.212 & 0.110 & 0.010 & 0.093 & -0.014 & 0.208 \\
Age 50 and above & $(0.108)$ & $(0.061)$ & $(0.825)$ & $(0.088)$ & $(0.795)$ & $(0.000)$ \\
years & 0.065 & 0.087 & 0.105 & 0.076 & 0.154 & 0.149 \\
Burundi & $(0.767)$ & $(0.497)$ & $(0.430)$ & $(0.656)$ & $(0.335)$ & $(0.275)$ \\
& -0.209 & 0.413 & 0.389 & 0.401 & 0.411 & 0.405 \\
Kenya & $(0.016)$ & $(0.000)$ & $(0.000)$ & $(0.000)$ & $(0.000)$ & $(0.000)$ \\
& 0.553 & 0.368 & 0.423 & 0.377 & 0.639 & 0.391 \\
Rwanda & $(0.000)$ & $(0.000)$ & $(0.000)$ & $(0.000)$ & $(0.000)$ & $(0.000)$ \\
& 0.305 & 0.437 & -0.416 & 0.437 & -0.425 & 0.416 \\
Tanzania & $(0.002)$ & $(0.000)$ & $(0.000)$ & $(0.000)$ & $(0.000)$ & $(0.000)$ \\
& 0.662 & 0.350 & 0.370 & 0.384 & 0.634 & 0.360 \\
Uganda & $(0.008)$ & $(0.000)$ & $(0.000)$ & $(0.000)$ & $(0.000)$ & $(0.000)$ \\
Cut 1 & 0.335 & 0.376 & 0.384 & 0.384 & 0.382 & 0.377 \\
Constant & 0.000 & $(0.000)$ & $(0.000)$ & $(0.000)$ & $(0.000)$ & $(0.000)$ \\
Cut 2 & 0.479 & 0.523 & -0.630 & 0.969 & 0.439 & 0.602 \\
Constant & 0.000 & $(0.000)$ & $(0.000)$ & $(0.000)$ & $(0.000)$ & $(0.000)$ \\
Log-likelihood & 0.815 & 0.898 & 0.515 & 0.585 & 0.823 & 0.978 \\
LR Chi-Square & 0.000 & $(0.000)$ & $(0.000)$ & $(0.000)$ & $(0.000)$ & $(0.000)$ \\
$\mathrm{R}^{2}$ - P-value & 508.875 & 538.662 & 565.186 & 488.721 & 538.617 & 533.228 \\
\hline Sourc: Aun & 139.630 & 74.607 & 59.284 & 59.007 & 58.669 & 52.214 \\
& 0.023 & 0.038 & 0.030 & 0.030 & 0.030 & 0.027 \\
\hline
\end{tabular}

Source: Author's calculations based on WBES data.

Table 6 confirms the findings that larger firms, foreign owned firms, publicly listed companies, and firms aged more than 10 years report lower financing obstacles as according to the regression results for specific financing obstacles on firm characteristics. The study report results using size dummies as size indicators and country dummies as country indicators. SMEs operates in both manufacturing and service sectors; operate locally; unincorporated [operate as family owned business (sole proprietorship) or partnership]; their growth are in early stage (less than 10 years) because most of they fail before 5 years and even if succeeded above 5 years they are stagnant because of shortage of finance to support profitable investment opportunities and growth.

Overall, the results in Table 4-6 consistently point the small and domestically owned firms are facing higher obstacles than other firms. The results show that domestic firms face higher obstacles than foreign firms is consistent with Harrison and McMillan (2003) who find that the investment-cash flow sensitivity is higher for domestic firms than foreign firms. Study findings demonstrate sorting firms according to their size and ownership structure (foreign vs. domestic ownership) in order to test the effect of financing obstacles leads to a reasonable classifications. This also suggests that classification criteria based on size and ownership are most useful in testing the presence of financing constraints and identifying financing constrained firms.

\section{Conclusion}

SMEs are the core to solve unemployment problem which is growing in East African countries. However, SMEs still have reported acute problems of access to external finance. This study explores the firm characteristics that predict best firms' financing obstacles. Survey on access to finance by World Bank survey, this study was conducted to assess indicators of financing constraints to SMEs. The study explores what can determine financing constraint to a firm by assessing firm characteristics: firm age, firm size, incorporation, type of ownership, sector of economic activity or country. The study find out that most of the firms experiencing financing obstacles tended to possess SMEs' features i.e. small and medium, young, sole proprietorship and 
partnership mostly operating in manufacturing and service industrial sectors. More studies might be conducted in this field as a panel structure of the survey where individual firms can be followed over time to provide a new dimension for research to improve SMEs financing obstacles.

\section{References}

Abor, J. (2008). Determinants of the capital structure of Ghanaian firms. Retrieved from http://www.aercafrica.org/documents/rp176.pdf

Aghion, P., Fally, T., \& Scarpetta, S. (2007). Credit constraints as a barrier to the entry and post-entry growth of firms. Economic Policy, 22, 731-779. http://dx.doi.org/10.1111/j.1468-0327.2007.00190.x

Artola, C., \& Genre, V. (2011). Euro area SMEs under financial constraints: belief or reality? European Central Bank, Kaiserstrasse 29, D-60311 Frankfurt, Germany. Retrieved from www.ecb.int/pub/pdf/other/financialstabilityreview201106en.pdf

Atanasova, C., \& Wilson, N. (2004). Disequilibrium in the UK corporate loan market. Journal of Banking and Finance, 28, 595-614. http://dx.doi.org/10.1016/S0378-4266(03)00037-2

Atieno, R. (2009). Linkages, access to finance and the performance of small-scale enterprises in Kenya. Journal of Accounting and Business Research, 3(1), 33-48.

Ayyagari, M., Beck, T., \& Demirgüç-Kunt, A. (2007). Small and medium enterprises across the globe. Small Business Economics, 29(4), 415-434. http://dx.doi.org/10.1007/s11187-006-9002-5

Balling, M., Bernet, B., \& Gnan, E. (2009). Financing SMEs in Europe. Four papers by: Rym Ayadi; Beat Bernet and Simone Westerfeld; Tom Franck and Nancy Huyghebaert; Vitor Gaspar, Simona Bovha-Padilla and Reinhilde Veugelers. SUERF-The European Money and Finance Forum; Vienna. Retrieved from http://www.suerf.org/download/studies/study20093.pdf

Bebczuk, R. N. (2004). What determines the Access to Credit by SMEs in Argentina? Documento de Trabajo Nro 48. Retrieved from http://www.depeco.econo.unlp.edu.ar/doctrab/doc48.pdf

Beck, T., Demirgüç-Kunt, A., \& Maksimovic, V. (2005). Financial and legal constraints to growth: does firm size matter? Journal of Finance, 60(1), 137-177. http://dx.doi.org/10.1111/j.1540-6261.2005.00727.x

Beck, T., Demirgüç-Kunt, A., Laeven, L., \& Maksimovic, V. (2006). The determinants of financing obstacles. Journal of International Money and Finance, 25, 932-952. http://dx.doi.org/10.1016/j.jimonfin.2006.07.005

Berger, A., \& Udell, G. (2006). A more conceptual framework for SME financing. Journal of Banking and Finance, 30(11), 2945-2966. http://dx.doi.org/10.1016/j.jbankfin.2006.05.008

Bougheas, S., Mizen, P., \& Yalcin, Y. (2006). Access to external financing: theory and evidence on the impact of monetary policy and firm-specific characteristics. Journal of Banking and Finance, 30, 199-227. http://dx.doi.org/10.1016/j.jbankfin.2005.01.002

Canton, E., Grilo, I., Monteagudo, J., \& Van Der Zwan, P. (2010). Investigating the perceptions of credit constraints in the European Union. ERIM Report Series, No ERS-2010-001-ORG. Retrieved from http://hdl.handle.net/1765/17699

Cassar, G. (2004). The financing of business start-ups. Journal of Business Venturing, 19(2), 261-283. http://dx.doi.org/10.1016/S0883-9026(03)00029-6

Coluzzi, C., Ferrando, A., \& Martinez-Carrascal, C. (2009). Financing obstacles and growth: an analysis for euro area non-financial corporations. ECB Working Paper No. 997. Retrieved from www.ecb.int/pub/pdf/scpwps/ecbwp997.pdf

Devereux, M., \& Schiantarelli, F. (1989). Investment, Financial Factors and Cash Flow: Evidence from UK Panel Data. NBER Working Papers No.3116. Retrieved from http://www.nber.org/papers/w3116.pdf

Diamond, D. W. (1984). Financial Intermediation and Delegated Monitoring. The Review of Economic Studies, 51(3), 393-414. http://dx.doi.org/10.2307/2297430

European Commission Enterprise and Industry. (2009). Cyclicality of SME finance: Literature survey, data analysis and econometric analysis. Arjan Ruis (EIM), André van Stel (EIM), Achilleas Tsamis (CSES), Wim Verhoeven (EIM) and Mark Whittle (CSES), Zoetermeer, 23 March 2009.

Fatoki, O., \& Asah, F. (2011). The impact of firm and entrepreneurial characteristics on access to debt finance by SMEs in King Williams' Town, South Africa. International Journal of Business and Management, 6(8). http://dx.doi.org/10.5539/ijbm.v6n8p170 
Fazzari, S. G., Hubbard, G., \& Petersen, B. (1988). Financing constraints and corporate investment. Brookings Papers on Economic Activity, 2, 141-195. http://dx.doi.org/10.2307/2534426

Ferrando, A., \& Griesshaber, N. (2011). Financing obstacles among euro area firms: who suffers most? ECB Working Paper No. 1293. Retrieved from http://www.ecb.int/pub/pdf/scpwps/ecbwp997.pdf

Field, A. (2005). Discovering Statistics Using SPSS (2nd ed.). London: Sage Publications.

Gilchrist, S., \& Himmelberg, C. (1995). Evidence on the role of cash flow for investment. Journal of Monetary Economics, 36, 541-572. http://dx.doi.org/10.1016/0304-3932(95)01223-0

Hall, G., Hutchinson, P., \& Michealas, N. (2000). Industry effect on the determinants of Unquoted SMEs Capital Structure. International Journal of the Economic of Business, 7(3), 297-312. http://dx.doi.org/10.1080/13571510050197203

Harrison, A., \& McMillan, M. (2003). Does direct foreign investment affect domestic credit constraints? Journal of International Economics, 61(1), 73-100. http://dx.doi.org/10.1016/S0022-1996(02)00078-8

Irwin, D., \& Scott, J. M. (2010). Barriers faced by SMEs in raising bank finance. International Journal of Entrepreneurial Behaviour \& Research, 16(3), 245-259. http://dx.doi.org/10.1108/13552551011042816

Kaplan S. N., \& Zingales, L. (2000). Investment-cash flow sensitivities are not valid measures of financing constraints. Quarterly Journal of Economics, 115, 707-712. http://dx.doi.org/10.1162/003355300554782

Kaplan, S. N., \& Zingales, L. (1997). Do investment-cash flow sensitivities provide useful measures of finance constraints? Quarterly Journal of Economics, 112, 169-215. http://dx.doi.org/10.1162/003355397555163

Kira, A. R., \& He, Z. (2012). The impact of firm characteristics in access of financing by small and medium-sized enterprises in Tanzania. International Journal of Business and Management, 7(24), 108-119. http://dx.doi.org/10.5539/ijbm.v7n24p108

Kuzilwa, J. A. (2005). The role of credit for small business success: A study of National Entrepreneurship Development Fund in Tanzania. Journal of Entrepreneurship, $14(2)$. http://dx.doi.org/10.1177/097135570501400204

Le, T. B. N., Venkatesh, S., \& Nguyen, V. T. (2006). Getting bank financing: study of Vietnamese private firms. Asia Pacific Journal of Management, 23(2), 209-227. http://dx.doi.org/10.1007/s10490-006-7167-8

Levy, B. (1993). Obstacles to Developing Indigenous Small and Medium Enterprises: An Empirical Assessment. The World Bank Economic Review, 7(1), 65-83. http://dx.doi.org/10.1093/wber/7.1.65

Maina, L. W. (2006). Kenya research findings and conclusions. African Media Development Initiative, 1-86.

Modigliani, F., \& Miller, M. H. (1963). Corporate income taxes and the cost of capital: A correction. American Economic Review, 53(3), 433-444.

Myers, S. C. (1984). Capital structure puzzle. Journal of Finance, 39(1), 575-592. http://dx.doi.org/10.2307/2327916

Myers, S. C., \& Majluf, N. S. (1984). Corporate financial and investment decisions when firms have information investors do not have. Journal of Financial Economics, 13, 187-221. http://dx.doi.org/10.1016/0304-405X(84)90023-0

Nachman, D., \& Noe, T. (1994). Optimal design of securities under asymmetric information. Review of Financial Studies, 7(1), 1-44. http://dx.doi.org/10.1093/rfs/7.1.1

Oreoluwa, A. R. (2011). Small and medium scale enterprises and economic growth in Nigeria: An assessment of financing options. Pakistan Journal of Business and Economic Review, 2(1). Retrieved from http://www.journalsbank.com/pjber_2_2.pdf

Peel, M. J., \& Wilson, N. (1996). Working capital and financial management practices in the small firms sector. International Small Business Journal, 14(2), 52-68. http://dx.doi.org/10.1177/0266242696142004

Pindyck, R. S., \& Rubinfeld, D. L. (1998). Econometric Models and Economic Forecasts (4th ed.). McGraw-Hill China Machine Press, Peoples Republic of China.

Saito, K., \& Villaneuva, D. (1981). Transactions cost of credit to small scale sector in the Philippines. Economic Development and Cultural Change, 29, 631-40. http://dx.doi.org/10.1086/451275

Shridhar, M. (2006). Incubators and science parks: strategies for developing countries. Retrieved from http://www.anprotec.org.br/idisc/pdfs/publiacoes/155.doc 
Slotty, C. F. (2009). Financial constraints and the decision to lease - Evidence from German SMEs. Goethe University Frankfurt, House of Finance, Germany. Retrieved from http://www.econstor.eu/dspace/bitstream/10419/39049/1/609306316.pdf

Stansfield, M., \& Grant, K. (2003). An investigation into issues influencing the use of the Internet and electronic commerce among small-medium sized enterprises. Journal of Electronic Commerce Research, 4(1), 15-33.

Townsend, R. M. (1979). Optimal Contracts and Competitive Markets with Costly State Verification. Journal of Economic Theory, 21, 265-293. http://dx.doi.org/10.1016/0022-0531(79)90031-0

Whited, T. M., \& Wu, G. (2006). Financial constraints risk. Review of Financial Studies, 19(2), 531-559. http://dx.doi.org/10.1093/rfs/hhj012

Wooldridge, J. M. (2009). Introductory Econometrics: A Modern Approach (4th ed.). Cengage Learning. Tsinghua University Press, People's Republic of China.

Yongqiang, L. Armstrong, A., \& Clarke, A. (2012). An instrument variable model of the impact of financing decisions on performance of small businesses in Australia's Pre-global Financial Crisis. Journal of Modern Accounting and Auditing, 8(7), 1052-1065. Victoria University, Melbourne, Australia.

\section{Appendix}

Appendix 1. Number of firms by country

\begin{tabular}{lcc}
\hline Country & No. of Firms & Percent \\
\hline 1. Burundi & 242 & 12.5 \\
2. Kenya & 657 & 34.0 \\
3. Rwanda & 158 & 8.2 \\
4. Tanzania & 368 & 19.0 \\
5. Uganda & 508 & 26.3 \\
Total & 1933 & 100 \\
\hline
\end{tabular}

Source: Author's calculations based on WBES data

Appendix 2. Variables definitions and source

\begin{tabular}{|c|c|c|c|}
\hline Variables & $\begin{array}{l}\text { Abbreviation } \\
\text { of Variables }\end{array}$ & Definition & Source \\
\hline Manufacturing & Manuf & $\begin{array}{l}\text { Dummy variable that takes on value } 1 \text { if a firm } \\
\text { operates in Manufacturing sector, otherwise } 0 .\end{array}$ & $\begin{array}{l}\text { The World Bank } \\
\text { Enterprise Survey }\end{array}$ \\
\hline Service & Serv & $\begin{array}{l}\text { Dummy variable that takes on value } 1 \text { if a firm } \\
\text { operates in Service sector, otherwise } 0 .\end{array}$ & $\begin{array}{l}\text { The World Bank } \\
\text { Enterprise Survey }\end{array}$ \\
\hline Construction & Const & $\begin{array}{l}\text { Dummy variable that takes on value } 1 \text { if a firm } \\
\text { operates in Construction sector, otherwise } 0 \text {. }\end{array}$ & $\begin{array}{l}\text { The World Bank } \\
\text { Enterprise Survey }\end{array}$ \\
\hline Small & Small & $\begin{array}{l}\text { Dummy variable that takes on value } 1 \text { if a firm is } \\
\text { Small enterprise, otherwise } 0 \text {. }\end{array}$ & $\begin{array}{l}\text { The World Bank } \\
\text { Enterprise Survey }\end{array}$ \\
\hline Medium & Medium & $\begin{array}{l}\text { Dummy variable that takes on value } 1 \text { if a firm is } \\
\text { Medium enterprise, otherwise } 0 \text {. }\end{array}$ & $\begin{array}{l}\text { The World Bank } \\
\text { Enterprise Survey }\end{array}$ \\
\hline Large & Large & $\begin{array}{l}\text { Dummy variable that takes on value } 1 \text { if a firm is } \\
\text { Large enterprise, otherwise } 0 .\end{array}$ & $\begin{array}{l}\text { The World Bank } \\
\text { Enterprise Survey }\end{array}$ \\
\hline Public Ltd Company & Plc & $\begin{array}{l}\text { Dummy variable that takes on value } 1 \text { if a firm is } \\
\text { Public Limited Company, otherwise } 0 \text {. }\end{array}$ & $\begin{array}{l}\text { The World Bank } \\
\text { Enterprise Survey }\end{array}$ \\
\hline $\begin{array}{l}\text { Private Ltd } \\
\text { Company }\end{array}$ & Pltdcoy & $\begin{array}{l}\text { Dummy variable that takes on value } 1 \text { if a firm is } \\
\text { Private held Limited Company, otherwise } 0 \text {. }\end{array}$ & $\begin{array}{l}\text { The World Bank } \\
\text { Enterprise Survey }\end{array}$ \\
\hline Sole Proprietorship & Solepro & $\begin{array}{l}\text { Dummy variable that takes on value } 1 \text { if a firm is a } \\
\text { Sole proprietorship, otherwise } 0 \text {. }\end{array}$ & $\begin{array}{l}\text { The World Bank } \\
\text { Enterprise Survey }\end{array}$ \\
\hline
\end{tabular}




\begin{tabular}{|c|c|c|c|}
\hline Partnership & Partnerp & $\begin{array}{l}\text { Dummy variable that takes on value } 1 \text { if a firm is } \\
\text { Partnership, otherwise } 0 \text {. }\end{array}$ & $\begin{array}{l}\text { The World Bank } \\
\text { Enterprise Survey }\end{array}$ \\
\hline Less than $5 \mathrm{yrs}$ & Age 5 & $\begin{array}{l}\text { Dummy variable that takes on value } 1 \text { if a firm is } \\
\text { Less than } 5 \text { years, otherwise } 0 .\end{array}$ & $\begin{array}{l}\text { The World Bank } \\
\text { Enterprise Survey }\end{array}$ \\
\hline From 5 to $9 \mathrm{yrs}$ & Age_5to9 & $\begin{array}{l}\text { Dummy variable that takes on value } 1 \text { if a firm is } \\
\text { from } 5 \text { years to } 9 \text { years, otherwise } 0 .\end{array}$ & $\begin{array}{l}\text { The World Bank } \\
\text { Enterprise Survey }\end{array}$ \\
\hline From 10 to $19 \mathrm{yrs}$ & Age_10to19 & $\begin{array}{l}\text { Dummy variable that takes on value } 1 \text { if a firm is } \\
\text { from } 10 \text { years to } 19 \text { years, otherwise } 0 .\end{array}$ & $\begin{array}{l}\text { The World Bank } \\
\text { Enterprise Survey }\end{array}$ \\
\hline From 20 to $49 \mathrm{yrs}$ & Age_20to49 & $\begin{array}{l}\text { Dummy variable that takes on value } 1 \text { if a firm is } \\
\text { from } 20 \text { years to } 49 \text { years, otherwise } 0 .\end{array}$ & $\begin{array}{l}\text { The World Bank } \\
\text { Enterprise Survey }\end{array}$ \\
\hline Above 50 yrs & Age_50 & $\begin{array}{l}\text { Dummy variable that takes on value } 1 \text { if a firm is } \\
\text { above } 50 \text { years and above, otherwise } 0 \text {. }\end{array}$ & $\begin{array}{l}\text { The World Bank } \\
\text { Enterprise Survey }\end{array}$ \\
\hline Private (Local) & PvtLC & $\begin{array}{l}\text { Dummy variable that takes on value } 1 \text { if a firm is } \\
\text { private locally owned, otherwise } 0 \text {. }\end{array}$ & $\begin{array}{l}\text { The World Bank } \\
\text { Enterprise Survey }\end{array}$ \\
\hline Private (Foreign) & PvtFG & $\begin{array}{l}\text { Dummy variable that takes on value } 1 \text { if a firm is } \\
\text { private foreign owned, otherwise } 0 .\end{array}$ & $\begin{array}{l}\text { The World Bank } \\
\text { Enterprise Survey }\end{array}$ \\
\hline State/Government & State & $\begin{array}{l}\text { Dummy variable that takes on value } 1 \text { if a firm is } \\
\text { state/government owned, otherwise } 0 \text {. }\end{array}$ & $\begin{array}{l}\text { The World Bank } \\
\text { Enterprise Survey }\end{array}$ \\
\hline General & Gcfin & $\begin{array}{l}\text { How financing obstacle affect your business } \\
\text { operations and firm growth: (1) No obstacle (2) } \\
\text { Minor obstacle (3) Moderate Obstacle (4) Major } \\
\text { obstacle (5) Very severe obstacle }\end{array}$ & $\begin{array}{l}\text { The World Bank } \\
\text { Enterprise Survey }\end{array}$ \\
\hline Collateral & FcColl & $\begin{array}{l}\text { Financing constraint collateral: (1) No obstacle (2) } \\
\text { Minor obstacle (3) Moderate Obstacle (4) Major } \\
\text { obstacle (5) Very severe obstacle }\end{array}$ & $\begin{array}{l}\text { The World Bank } \\
\text { Enterprise Survey }\end{array}$ \\
\hline $\begin{array}{l}\text { Application } \\
\text { Procedures }\end{array}$ & FcApp & $\begin{array}{l}\text { Financing constraint application procedures (1) } \\
\text { No obstacle (2) Minor obstacle (3) Moderate } \\
\text { Obstacle (4) Major obstacle (5) Very severe } \\
\text { obstacle }\end{array}$ & $\begin{array}{l}\text { The World Bank } \\
\text { Enterprise Survey }\end{array}$ \\
\hline High Interest rates & FcHInte & $\begin{array}{l}\text { Financing constraint high interest rate (1) No } \\
\text { obstacle (2) Minor obstacle (3) Moderate Obstacle } \\
\text { (4) Major obstacle (5) Very severe obstacle }\end{array}$ & $\begin{array}{l}\text { The World Bank } \\
\text { Enterprise Survey }\end{array}$ \\
\hline Credit & FcCredit & $\begin{array}{l}\text { Financing constraint credit accessibility (1) No } \\
\text { obstacle (2) Minor obstacle (3) Moderate Obstacle } \\
\text { (4) Major obstacle (5) Very severe obstacle }\end{array}$ & $\begin{array}{l}\text { The World Bank } \\
\text { Enterprise Survey }\end{array}$ \\
\hline $\begin{array}{l}\text { Corruption\& Special } \\
\text { Connections }\end{array}$ & FcConr & $\begin{array}{l}\text { Financing constraint special connections and } \\
\text { corruption (1) No obstacle (2) Minor obstacle ( } 3 \text { ) } \\
\text { Moderate Obstacle (4) Major obstacle (5) Very } \\
\text { severe obstacle }\end{array}$ & $\begin{array}{l}\text { The World Bank } \\
\text { Enterprise Survey }\end{array}$ \\
\hline $\begin{array}{l}\text { Loan Size \& } \\
\text { Maturity }\end{array}$ & FcSizMat & $\begin{array}{l}\text { Financing constraint Loan size and time Maturity } \\
\text { (1) No obstacle (2) Minor obstacle (3) Moderate } \\
\text { Obstacle (4) Major obstacle (5) Very severe } \\
\text { obstacle }\end{array}$ & $\begin{array}{l}\text { The World Bank } \\
\text { Enterprise Survey }\end{array}$ \\
\hline
\end{tabular}

\section{Notes}

Note 1. East African Countries comprise Burundi, Kenya, Rwanda, Tanzania and Uganda.

Note 2. World Bank Enterprises Survey web: www.enterprisesurveys.org

Note 3. Figure 1 is adapted and updated from Carey et al. (1993, Figure 10) and Berger \& Udell (1998, Figure 1)

Note 4. See Brewer and Genay (1994) and Brewer et al. (1997) for empirical evidence that external equity in the 
form venture capital is more likely to be used to finance intangible assets and activities that generate little collateral while external private debt is more likely to be used to finance tangible assets.

Note 5. Venture capital is a broad term used to describe funding acquired in the earlier stages of a firm's economic life. This type of funding is usually acquired during the period which the company is growing faster than its ability to generate internal financing and before the company has achieved the size needed to be efficient.

Note 6. See Brewer and Genay (1994) and Brewer et al. (1997) for empirical evidence that external equity in the form venture capital is more likely to be used to finance intangible assets and activities that generate little collateral while external private debt is more likely to be used to finance tangible assets.

Note 7. This is not the only argument that has been suggested as the driving optimality of the type of equity contracts we observe in the venture capital, Garmaise (1997) argued that the normal pecking order in which external debt precedes external equity can be reserved if it is assumed that venture capitalists have superior information to entrepreneurs. While it seems plausible to argue that entrepreneurs have a superior informational advantage over a certain aspects of their project such as the feasibility of their project's technology, it may be reasonable to assume that venture capitalists have superior information over a project's marketability and its operational implementation..

Note 8. For example, in 2009 G-20 countries committed to identifying lessons learned on innovative approaches to providing financial services to SMEs and to promoting successful regulatory and policy approaches (Pittsburgh G-20 Summit, 2009). Also see OECD, 2004 \& 2008.

Note 9. The SMEs nomenclature in Tanzania is used to mean micro, small and medium enterprises. Micro enterprises are those engaging up to 4 people or employing capital of up to US\$5,000. Small enterprises have between 5 and 49 employees or capital of US\$5,000 to US\$200,000. Medium enterprises employ between 50 and 99 people or use capital investment from US\$200,000 to US\$ 800,000 .

Note 10. International Finance Company (IFC. 2005) - Tanzania MSMEs Access to Finance Assessment.

Note 11. This is especially true for businesses outside of the import/export sectors. A World Bank report in June 2008 noted that $64 \%$ of businesses in trade reported that credit was a significant constraint on their business. For manufacturing and agriculture, the corresponding figures were $86 \%$ and $88 \%$.

Note 12. See Rwanda Establishments Census, June 2011 Gross enrolment rate for secondary education increased from $20.7 \%$ in 2008 to $31.5 \%$ in 2010 with the share of higher education students in science and technology increasing from $21 \%$ to $41.4 \%$ during the same period. Access to Technical and Vocational Education and Training (TVET) has increased only marginally from 8,250 students in 2006 to 15,354 in 2010, and remains well below the 2012 target of 135,000 students.

Note 13. African Development Bank; African Development Fund: Rwanda Bank Group Country Strategy Paper 2012-2016; Regional Department East A (OREA); October 2011: Task team: E. B. Sennoga (RWFO); N. Makonnen (RWFO);C. Baumont (OREA); S. Turay (OREA); O. Amu (RWFO); E. Ferreras Carreras (ORQR.4); M. Mdachi (OSHD.2); G. Ajumbo (ONRI.2); C. Mollinedo (OSGE.2); E. Ngode (ORPF.2); J.P. Kayobotsi (RWFO); J. Karimba (RWFO); J. Nyirimana (RWFO); S. Okeke (OSAN.1); E. Zeleke (ORQR.3); E. Rutaboba (RWFO); P. Munyaruyenzi (RWFO); and B. Byamukama (RWFO) ; Peer Reviewers: R. Walker (KEFO); P. Kariuki (UGFO); J.C. Anyanwu (EDRE.1); S. Ijeh (ORWA); K. Mbekeani (ONRI.2); and S. Jean (OPSM).

Note 14. Kaplan and Zingales (1997, 2000) questioned the validity of Fazzary et al.'s findings that financially constrained firms tend to have high investment-cash flow sensitivity arguing that Fazzari et al. tends to classify firms incorrectly. This stream of the literature, using balance sheet information, needs to a priori classify firms between financially constrained and unconstrained firms (using proxies such as the size or the age of the firm) in order to check whether the sensitivity of investment/growth to cash-flow is higher for constrained than for unconstrained firms Kaplan and Zingales, after re-classification, find substantial differences in the degree of investment sensitivity to financial constraints between firms.

Note 15. The data source is the Enterprise Surveys database maintained by World Bank and freely available at www.enterprisesurveys.org 